\title{
Staying InformED: Top emergency Medicine pharmacotherapy articles of 2020
}

\author{
David E. Zimmerman, PharmD ${ }^{\mathrm{a}, *}$, Preeyaporn Sarangarm, PharmD ${ }^{\mathrm{b}}$, Caitlin S. Brown, PharmD ${ }^{\mathrm{c}}$, \\ Brett Faine, PharmD, MS ${ }^{\mathrm{d}}$, Tara Flack, PharmD ${ }^{\mathrm{e}}$, Brian W. Gilbert, PharmD ${ }^{\mathrm{f}}$, Gavin T. Howington, PharmD ${ }^{\mathrm{g}, \mathrm{h}}$, \\ Gregory Kelly, PharmD ${ }^{\mathrm{i}}$, Jessica Laub, PharmD ${ }^{\mathrm{j}}$, Blake A. Porter, PharmD ${ }^{\mathrm{k}}$, \\ Giles W. Slocum, PharmD ${ }^{1}$, Megan A. Rech, PharmD, MS ${ }^{\text {m,n }}$
}

\footnotetext{
a Duquesne University School of Pharmacy, University of Pittsburgh Medical Center-Mercy Hospital, Room 311 Bayer Learning Center, 600 Forbes Avenue, Pittsburgh, PA 15282, United States of America

${ }^{\mathrm{b}}$ Department of Pharmacy, University of New Mexico Hospital, Albuquerque, NM 87106, United States of America

c Department of Pharmacy, Mayo Clinic, 200 First Street SW, Rochester, MN 55905, United States of America

d Department of Emergency Medicine and Pharmacy Practice, University of Iowa, Iowa City, IA 52242, United States of America

e Department of Pharmacy, IU Health Methodist Hospital, Indianapolis, IN 46202, United States of America

${ }^{\mathrm{f}}$ Department of Pharmacy, Wesley Medical Center, Wichita, KS 67205, United States of America

${ }^{g}$ Department of Pharmacy Practice and Science, University of Kentucky College of Pharmacy, Lexington, KY 40506, United States of America

h Department of Pharmacy Services, University of Kentucky HealthCare, Lexington, KY 40536, United States of America

i Department of Pharmacy, Hospital of the University of Pennsylvania, Philadelphia, PA 19104, United States of America

${ }^{j}$ Department of Pharmacy, New York-Presbyterian-Brooklyn Methodist Hospital, Brooklyn, NY 11215, United States of America

${ }^{\mathrm{k}}$ Department of Pharmacy, University of Vermont Medical Center, Burlington, VT 05401, United States of America

${ }^{1}$ Department of Emergency Medicine and Department of Pharmacy, Rush University Medical Center, Chicago, IL 60612, United States of America

m Loyola University Chicago, Loyola University Medical Center, Stritch School of Medicine, Department of Emergency Medicine, S 1st Ave, Maywood 60153, IL, United States of America

${ }^{\mathrm{n}}$ Loyola University Medical Center, Department of Pharmacy, S 1st Ave, Maywood, IL 60153, United States of America
}

\section{A R T I C L E I N F O}

\section{Article history:}

Received 17 April 2021

Received in revised form 14 May 2021

Accepted 24 May 2021

\section{Keywords:}

Emergency medicine

Pharmacotherapy

Review

\begin{abstract}
A B S T R A C T
The year 2020 was not easy for Emergency Medicine (EM) clinicians with the burden of tackling a pandemic. A large focus, rightfully so, was placed on the evolving diagnosis and management of patients with COVID-19 and, as such, the ability of clinicians to remain up to date on key EM pharmacotherapy literature may have been compromised. This article reviews the most important EM pharmacotherapy publications indexed in 2020. A modified Delphi approach was utilized for selected journals to identify the most impactful EM pharmacotherapy studies. A total of fifteen articles, eleven trials and four meta-analyses, were identified. This review provides a summary of each study, along with a commentary on the impact to the EM literature and EM clinician.
\end{abstract}

(C) 2021 Elsevier Inc. All rights reserved.

\section{Introduction}

Emergency medicine (EM) literature expands every year, and 2020 was no exception. EM clinicians must stay current with a wide variety of disease states and treatments spanning the breadth of ambulatory care to critical care. As such, the constant flow of new publications presents a significant challenge to EM clinicians to keep up with evidencebased practices, which was likely compounded in 2020 by the novel coronavirus (COVID-19) pandemic. In order to address this issue for EM clinicians, the Emergency Medicine PHARMacotherapy research NETwork (EMPHARM-NET), set to identify, summarize, and apply the most impactful EM articles of 2020 pertaining to pharmacotherapy.

\footnotetext{
* Corresponding author.

E-mail address: Zimmerm6@duq.edu (D.E. Zimmerman).
}

EMPHARM-NET is a network of geographically and demographically diverse EM clinical pharmacist researchers that was formed in 2019 with the goal of fostering and conducting high quality, transformative, multidisciplinary research pertaining pharmacotherapy in the emergently ill population across the United States.

Using a modified Delphi approach, authors were divided into pairs to review the 2020 table of contents for selected journals deemed relevant to EM practice (Appendix A) with the purpose of evaluating adult, nonCOVID-19-related literature using the Grading of Recommendations, Assessment, Development and Evaluation (GRADE) system [1]. The GRADE system is a validated and objective tool used to evaluate the quality of published articles into four levels: high, moderate, low, and very low [1]. The Delphi approach was utilized as a way to systematically and objectively review the published literature and come to a consensus as a group, as has been used previously [2]. The list of journals 
being reviewed was reviewed and agreed upon by the research team by group consensus. Articles pertaining to COVID-19 were excluded from this review due to high volume and the rapidly changing nature of pertinent literature. In reviewing non-EM specialty journals (e.g. critical care, cardiology, and infectious disease), an emphasis was placed on literature where care was initiated and managed in the emergency department (ED). Articles deemed to be GRADE 1A or 1B were selected for potential inclusion. In the event of discordance between two reviewers, adjudication was performed by the primary author (DEZ). A total of 27 articles met GRADE 1A or 1B and were collectively reviewed by the research team. A total of ten studies and four meta-analyses were included in this review based upon the consensus of the group on their impact on EM practice.

Effect of Ascorbic Acid, Corticosteroids, and Thiamine on Organ Injury in Septic Shock: The ACTS Randomized Clinical Trial [3] [GRADE 1A].

Effect of Vitamin C, Hydrocortisone, and Thiamine vs. Hydrocortisone Alone on Time Alive and Free of Vasopressor Support Among Patients With Septic Shock: The VITAMINS Randomized Clinical Trial [4] [GRADE $1 \mathrm{~A}]$.

Interest in hydrocortisone, ascorbic acid, and thiamine ("HAT" therapy) continues to grow following a single center interventional study that found a significant in-hospital mortality benefit, mortality rate of $8.5 \%$ in the treatment vs. $40.4 \%$ in the control group, amongst patients with severe sepsis or septic shock [5]. The study yielded impressive findings but had significant limitations warranting further exploration through randomized, controlled trials (RCTs). Two RCTs were published in 2020 in response to these initial findings. The first was the effect of ascorbic acid, corticosteroids, and thiamine on organ injury in septic shock (ACTS) trial, a multicenter, blinded, placebo-controlled study that randomized 205 patients to $1500 \mathrm{mg}$ intravenous (IV) ascorbic acid, $50 \mathrm{mg}$ IV hydrocortisone, and $100 \mathrm{mg}$ IV thiamine every $6 \mathrm{~h}$ for four days or until intensive care unit (ICU) discharge or placebo [3]. The primary outcome of change in Sequential Organ Failure Assessment (SOFA) score between enrollment and 72-h follow-up was similar between groups ( -4.7 intervention vs. -4.1 in placebo) (adjusted mean difference of $-0.8 ; 95 \% \mathrm{CI}-1.7$ to 0.2 ). No differences were observed in mortality or other exploratory secondary outcomes except for shock-free days (five in intervention group vs. four in placebo group; median difference of 1 day; $95 \% \mathrm{CI} 0.2$ to 1.8 ).

The VITAMINS Trial was a multicenter, open-label, RCT in 211 patients presenting with Sepsis-3 defined septic shock throughout 10 ICUs in Australia, New Zealand, and Brazil [4]. The primary endpoint of total time patients were alive and vasopressor-free seven days postrandomization was similar between the intervention group and the hydrocortisone alone group (122.1 h intervention group [IQR 76.3-145.5 h] vs. $124.6 \mathrm{~h}$ placebo group [IQR $82.1-147.0 \mathrm{~h}$ ]). There was no difference in secondary outcomes between groups with respect to 28-day, 90-day, or ICU mortality.

Multiple limitations exist with both studies. In the ACTS trial, a very small number of patients were included, 205 out of 4569 screened due to the receipt of corticosteroids. While this exclusion allowed for effective placebo control, it limits the applicability of these results to a patient population that may be more critically ill. The ACTS trial only evaluated patients with septic shock in contrast to prior studies which included patients with severe sepsis and may not be applicable to patients with sepsis without shock. In both studies, there were time to HAT therapy initiation delays suggesting earlier pharmacologic intervention may be beneficial. In the VITAMINS study, the effect of thiamine and vitamin $C$ were not evaluated independently including safety and efficacy endpoints. Lastly, the authors did not report common septic shock standard of care confounders such as antibiotic administration and initial fluid requirements between groups. At this time, the use of
HAT therapy cannot be rountinely recommended in patients with sepsis and/or shock. Future studies are needed to address the impact of early HAT therapy administered in the ED on patient outcomes.

Tranexamic acid: Effect of Out-of-Hospital Tranexamic Acid vs. Placebo on 6-Month Functional Neurologic Outcomes in Patients with Moderate or Severe Traumatic Brain Injury [6] [GRADE 1A].

In 2010, the CRASH-2 trial showed an all cause mortality benefit (Relative Risk (RR) 0.91 [95\% CI 0.85 to 0.97]) for traumatic hemorrhage patients who received tranexamic acid (TXA) [7]. In 2019, the CRASH-3 trial investigated patients with traumatic brain injury (TBI) (originally excluded from the CRASH-2 trial) and found no statistical difference in head injury-related death between TXA and placebo [8]. However, the risk was reduced in a sub-group of patients with mild-to-moderate head injury (RR 0.78 [95\% CI 0.64-0.95]).

A recent double-blind, three-group, phase II RCT included 966 prehospital patients with moderate or severe blunt or penetrating TBI across the US and Canada [6]. The impact of TXA administered within two hours of injury on six-month neurologic outcomes in patients with moderate or severe TBI who were not in shock, defined as a systolic blood pressure of at least $90 \mathrm{mmHg}$, was evaluated. Patients were randomized to either a bolus maintenance group (TXA $1 \mathrm{~g}$ IV bolus, followed by TXA $1 \mathrm{~g}$ IV infusion over eight hours), bolus only group (TXA $2 \mathrm{~g}$ IV bolus, followed by placebo IV infusion), or a placebo group (IV bolus and infusion of placebo). All boluses were given in the prehospital setting and infusions were administered in-hospital.There was no difference in the primary outcome of favorable neurologic outcome (Glasgow Outcome Scale-Extended score $>4$ ) at six months between the combined TXA groups versus placebo. Of the patients included in the final analysis, 545 (56\%) showed intracranial hemorrhage (ICH) on baseline head CT, while 221 (23\%) had no injury. The authors completed an adjusted analysis by combining the two TXA regimens compared with placebo. A combined analysis comparing both TXA groups to placebo demonstrated $65 \%$ of those patients who received TXA had a favorable neurologic outcome, as compared with $62 \%$ in the placebo group but this was not statistically signficiant. Mortality at 28 days was $14 \%$ in the TXA group and $17 \%$ in the placebo group (adjusted difference $-2.9 \%$; $95 \% \mathrm{CI}-7.9 \%$ to $2.1 \%$ ). Thrombotic events occurred in $10 \%$ of the placebo group, $9 \%$ in the bolus only group, and $4 \%$ in the bolus maintenance group. Seizures were seen in $5 \%$ of the bolus only group, while only $2 \%$ in both bolus maintenance group and the placebo group. None of these values were statistically significant. TXA given within two hours of moderate or severe TBI did not improve the likelihood of favorable neurologic outcome or 28-day mortality. Regardless of the dosing regimen used, this study does not support the administration of pre-hospital TXA to improve mortality in patients with TBI.

Effects of High-Dose 24-h Infusion of Tranexamic Acid on Death and Thromboembolic Events in Patients with Acute Gastrointestinal Bleeding (HALT-IT) [9] [GRADE 1A].

The HALT-IT trial was a large, international, RCT evaluating the role of TXA in gastrointestinal bleeds (GIB) [9]. Patients presenting with significant upper or lower GIB were randomized to either TXA $1 \mathrm{~g}$ IV bolus followed by TXA $3 \mathrm{~g}$ IV infusion over $24 \mathrm{~h}(n=5956)$ or identical placebo, $0.9 \%$ sodium chloride, regimen $(n=5981)$. The classification of significant GIB was based on clinical discretion of the prescriber and included risk of bleeding to death, signs of shock, likely requirement for transfusion, or urgent endoscopic or surgical intervention. The primary outcome was death due to bleeding within five days of randomization. Of note, this was changed from the primary endpoint of all-cause mortality at 28 days five years into the study as a majority of deaths were due to non-bleeding causes. Death due to bleeding within five days occurred in $3.7 \%$ of patients in the TXA group and $3.8 \%$ of patients in the 
placebo group ( $R R$ 0.99; 95\% CI 0.82 to 1.18). There was no difference in the secondary outcomes including the original primary outcome, except patients in the TXA group had a significantly higher number of venous thromboembolic events compared to placebo (RR 1.85 ; $95 \% \mathrm{CI} 1.15$ to 2.9) and seizures (RR 1.73; 95\% CI 1.03 to 2.93), perhaps owing to the larger dose of TXA administered over a longer period. The major limitation to this study was the average time of onset to randomization being 21.4 and $22.5 \mathrm{~h}$ for TXA and placebo, respectively. Additionally, the timing of the onset of GIB is difficult to determine with most patients presenting $>3 \mathrm{~h}$ after bleeding onset, likely limiting the impact of TXA on inhibiting fibrinolysis. Current evidence does not support the use of TXA in patients presenting with a GIB.

Ketamine Versus Etomidate and Peri-intubation Hypotension: A National Emergency Airway Registry Study [10] [GRADE 1B].

Etomidate Use Is Associated With Less Hypotension Than Ketamine for Emergency Department Sepsis Intubations: A NEAR Cohort Study [11] [GRADE 1B].

Etomidate and ketamine are two induction agents commonly used in the ED that have inspired controversary surrounding safety of use in critically ill patients requiring rapid sequence intubation (RSI) [12]. Etomidate is considered hemodynamically neutral, but is associated with adrenal insufficiency and potential risk of increased mortality amongst sepsis patients [13-15]. Ketamine lacks adrenal suppression and may augment blood pressure given its sympathomimetic effects, making it a frequently used alternative to etomidate [16,17]. However, studies have reported hypotension and cardiac arrest with ketamine, likely from catecholamine depletion as a result of a septic state $[18,19]$. Two cohort studies used the National Emergency Airway Registry (NEAR) database to determine the hemodynamic impact of ketamine versus etomidate in all ED patients requiring endotracheal intubation and compared adverse event rates in patients with sepsis $[10,11]$. Although they have a GRADE of 1B, these two studies evaluated a large real world database and bring new information to a vital EM topic where there are many unanswered questions.

The first NEAR database cohort study including normotensive patients greater than 14 years of age who underwent RSI in the ED and received either ketamine $(n=738)$ or etomidate $(n=6068)$ [10]. Periintubation hypotension occurred in $18.3 \%$ of ketamine versus $12.4 \%$ of etomidate encounters (absolute difference $-5.9 \%$; $95 \%$ CI 2.9 to $8.8 \%$ ). Peri-intubation hypotensive events required treatment in $15.4 \%$ of the ketamine versus $8.9 \%$ of etomidate encounters (absolute difference $6.5 \%$; $95 \%$ CI 3.9 to $9.3 \%$ ). A dose-dependent response in periintubation hypotension events was not found in either group.

The second cohort study evaluated the current use of induction agents in septic patients ( $n=531)$ requiring RSI in the ED [11]. Ketamine was utilized more frequently in sepsis compared to non-sepsis cases $(27.3 \%$ vs. $11.7 \%$, OR $=2.8 ; 95 \%$ CI 2.3 to 3.5$)$ and etomidate was used less in sepsis versus non-sepsis cases $(70.8 \%$ vs. $84.6 \%$, OR $=0.4 ; 95 \% \mathrm{CI} 0.4$ to 0.5 ). Rates of post-intubation hypotension were more common in the ketamine vs. etomidate group 74.1 vs. $49.5 \%$ $(\mathrm{OR}=2.9 ; 95 \% \mathrm{CI} 1.9$ to 4.5$)$. Interestingly, there was significant variability $(0-92 \%)$ in septic patients intubated with ketamine when stratified by study site.

To date, only one RCT has compared etomidate versus ketamine in critically ill patients requiring RSI. Unfortunately, rates of postintubation hypotensive events were not evaluated so a direct comparison with the findings from the NEAR studies cannot be made [20]. Findings from the NEAR studies should be considered preliminary but should cause pause for ED providers who recommend ketamine as an alternative to etomidate in the setting of hypotensive septic patients. A multi-center RCT is necessary to determine the safest induction agent for sepsis patients requiring emergency intubation.
Resuscitation Fluids in Septic Shock: A Network Meta-Analysis of Randomized Controlled Trials [21] [GRADE 1A).

Surviving Sepsis guidelines recommend $30 \mathrm{~mL} / \mathrm{kg}$ of IV crystalloid fluid within three hours of presentation for septic shock [22]. While increasing evidence suggests that balanced crystalloids may represent the safest option for fluid resuscitation, current guidelines do not differentiate between type of crystalloid fluid; however, guidelines recommend against use of hydroxyethyl starches (HES) or gelatin (GEL) [22]. A network meta-analysis (NMA) assessed resuscitation fluids in septic shock [21].

The NMA included 13 RCTs in adults with severe sepsis or septic shock and compared the effect of resuscitation fluids [21]. The primary outcomes were 28 and 90-day mortality. Secondary outcomes included acute kidney injury (AKI) and need for renal replacement therapy (RRT). The resuscitation fluids were classified as $0.9 \% \mathrm{NaCl}(\mathrm{NS})$, hypertonic saline-dextran (HS), balanced solutions (BS), hypertonic sodium chloride/hydroxyethyl starch 40 solution (HSH40), high-molecularweight (MW) HES (H-HES), low-MW HES (L-HES), GEL, and albumin (ALB). Surface under cumulative ranking (SUCRA) were performed to identify the best treatment.

Twenty-eight-day mortality was evaluated in 11 studies (6610 patients) with eight eligible comparisons. There was a significant difference between BS and NS. SUCRAs demonstrated that HSH40 ranked the highest (93.8\%), BS ranked second (69.6\%), and NS last (25.1\%). For 90-day mortality, five studies with five eligible comparisons (4177 patients) were evaluated with no significant differences between the groups. AKI incidence was reported in five studies with five comparisons (1733 patients). There were significantly lower rates of AKI with GEL, BS, and L-HES when compared to H-HES. SUCRAs demonstrated GEL was the best (74.4\%), NS was second (64.9\%), and BS was third (58.3\%). The need for RRT was evaluated in five studies and included five eligible comparisons (1696 patients). There was a statistically significant difference between BS and H-HES, NS and H-HES, and L-HES and BS. SUCRAs for the need for RRT revealed that NS ranked the first (91.6\%), followed by BS (74.4\%); however, this endpoint is difficult to interpret as only a subset of the studies included this datapoint. Evidence continues to support the use of BS as the preferred resuscitation fluid for septic shock based on safety and mortality outcomes. Further confirmatory studies are needed to improve overall fluid resuscitation strategies in sepsis patients.

Effect of Reduced Exposure to Vasopressors on 90-Day Mortality in Older Critically Ill Patients with Vasodilatory Hypotension: A Randomized Clinical Trial [23] [GRADE 1A].

A previous meta-analysis suggested targeting increased mean arterial pressure (MAP) in a subgroup of elderly patients on vasopressors may increase mortality [24]. Based on this finding, a pragmatic, unblinded, multicenter, randomized trial evaluated the utilization of vasopressors guided by permissive hypotension (MAP 60-65 $\mathrm{mmHg}$ ) or usual care in 65 ICUs in the United Kingdom [23]. Elderly patients ( $\geq$ 65 years old) presenting with vasodilatory shock within six hours of vasopressor initiation were included. Choice of vasopressor was determined by the treating clinician and doses were reported in norepinephrine equivalents. Norepinephrine, vasopressin, terlipressin, phenylephrine, epinephrine, dopamine, and metaraminol were considered as vasopressors. Overall, 2463 patients with similar baseline demographics who primarily received norepinephrine were included. There was no difference in 90-day mortality between the permissive hypotension vs. usual care groups (41.0\% vs. $43.8 \%$ ). ICU and hospital mortality were similar. However, after adjusting for pre-specified baseline characteristics, the 90-day mortality OR was 0.82 (95\% CI 0.68 to 0.98 ) favoring permissive hypotension. Patients in the permissive hypotension group also had reduced median exposure to vasopressors of $33 \mathrm{~h}$ vs. $38 \mathrm{~h}$, respectively (absolute difference $-5.0 ; 95 \% \mathrm{CI}-7.8$ to -2.2 ) and a 
reduced median total dose of vasopressors (absolute difference -8.7 $\mathrm{mg}$; $95 \% \mathrm{CI}-12.8$ to -4.6 ). Other outcomes, including length of stay and adverse events, were similar between groups. Overall, this welldesigned trial demonstrated a reduction in mortality after adjustment in elderly patients on vasopressors. In elderly patients with vasodilatory shock requiring vasopressors, targeting a lower MAP may be considered to improve clinical outcomes.

Survival After Intravenous Versus Intraosseous Amiodarone, Lidocaine, or Placebo in Out-of-Hospital Shock-Refractory Cardiac Arrest [25] [GRADE 1A].

The intraosseous (IO) route is used as alternative access when IV access cannot be obtained. Recently, trials have demonstrated worse outcomes with IO drug administration compared to IV in out-of-hospital cardiac arrest (OHCA), due to concerns with drug absorption and a low-flow state limiting circulation of medications [26-29]. While these studies are not without bias, this led to an update in the 2020 American Heart Association Basic and Advanced Cardiac Life Support guidelines to list IV as the favorable initial approach, but lists IO as an option when IV access is not available [30].

This study was a priori secondary analysis of a previously published randomized, double-blinded study of 55 Emergency Medicial Service (EMS) agencies from ten study sites in North America including 3026 patients randomized to amiodarone, lidocaine or placebo ( $0.9 \%$ sodium chloride) in shock-refractory ventricular fibrillation or ventricular tachycardia non-traumatic OHCA [31]. The primary outcome was survival difference between IV and IO administration of amiodarone and lidocaine versus placebo. Route of administration was at the discretion of EMS providers.

Approximately $78 \%$ of patients received the study medication intravenously. When amiodarone was compared to placebo, a survival benefit at hospital discharge was found when administered IV (absolute risk reduction (ARR) $1.23 ; 95 \% \mathrm{CI} 1.11$ to 1.37 ) but not when given IO (ARR 0.95; 95\% CI 0.75 to 1.21 ). Lidocaine showed similar survival to discharge benefit when administered IV (ARR 1.21; 95\% CI 1.02 to 1.45 ) but not IO (ARR 1.03 ; $95 \%$ CI 0.74 to 1.44 ) when compared to placebo. There were no differences in outcomes between IV and IO placebo.

As this study was a secondary analysis, it was not adequately powered to detect a survival difference between IV and IO administration routes, nor were patients randomized to route of administration. Given IV access is routinely attempted prior to IO administration there is likely selection bias for the patients in the IO group, including patients who are volume depleted and potentially have increased comorbidities. This limits the ability to impart a practice change to recommend against the use of IO administration. However, this does show the need for further research regarding optimal administration routes in OHCA.

Reappraisal of the treatment duration of antibiotic regimens for acute uncomplicated cystitis in adult women: a systematic review and network meta-analysis of 61 randomised clinical trials [32] [GRADE 1A].

Acute uncomplicated cystitis is a common pathology resulting in approximately two million female ED visits annually [33]. The 2010 Infectious Diseases Society of America and European Society for Clinical Microbiology and Infectious Diseases International Clinical Practice guidelines recommend nitrofurantoin, sulfamethoxazoletrimethoprim, and fosfomycin as five-day, three-day, and single-day regimens, respectively, despite evidence suggesting shorter treatment durations [34].

Kim et al. conducted a meta-analysis including trials evaluating female patients with acute uncomplicated cystitis, assessment of recommended antibiotic therapies, clinical or microbial response outcomes, and of RCT design [32]. Quality of evidence was determined by the GRADE approach [1].
Sixty-one prospective RCTs were analyzed ( $n=20,780)$. A threeday sulfamethoxazole-trimethoprim regimen was more effective than a single-dose regarding clinical response (RR 1.15; 95\% CI 1.01 to 1.31 ), but showed no difference in microbial response (RR 1.02; 95\% CI 0.95 to 1.10). Three-day second-generation fluoroquinolone regimens (e.g., ciprofloxacin) were more effective than a single-dose of the same antibiotic in clinical response (RR $1.04 ; 95 \% \mathrm{CI} 1.01$ to 1.08 ) and microbial response (RR 1.04; 95\% CI 1.003 to 1.08). There were no significant differences between three-day third-generation fluoroquinolone (e.g., levofloxacin) regimens versus a single-dose (RR 0.99; 95\% CI 0.94 to 1.05 and microbial response (RR 1.03; 95\% CI 0.96 to 1.11). There were no differences in clinical response between three-day third-generation cephalosporin regimens versus single-doses (RR $1.04 ; 95 \% \mathrm{CI} 0.85$ to 1.34 ) and seven-day third generation cephalosporin regimens versus single-doses ( $\mathrm{RR} 1.02 ; 95 \% \mathrm{CI} 0.75$ to 1.39 ). There were no differences in microbial response for three-day first and third generation cephalosporin regimens versus single doses (RR 1.33; 95\% CI 0.80 to 2.46 and RR $1.06 ; 95 \% \mathrm{CI} 0.88$ to 1.34 , respectively). Lastly, there was no difference between three-day amoxicillin-clavulanate regimens versus single-doses (clinical response RR 0.98 ; $95 \% \mathrm{CI} 0.88$ to 1.10 ) and microbial response (RR $0.99 ; 95 \% \mathrm{CI} 0.85$ to 1.14 ).

It may be reasonable for patients with a potentially self-limiting pathology to be treated with shorter antibiotic durations. However, given the quality of evidence evaluated over a large time frame, and the variation in antibiotics and dosing utilized across the included trials, durations supported by current practice guidelines should still be considered standard practice.

Effect of Oral Oseltamivir on Virological Outcomes in Low-risk Adults with Influenza: A Randomized Clinical Trial [35] [GRADE 1A].

Duration of viral shedding is thought to be a determinant of infectivity and transmissibility [36]. While the clinical efficacy of oseltamivir has previously been established in patients with influenza, data are limited on the virologic efficacy of oseltamivir. This study was a randomized, double-blind study conducted in Thailand, the United States, and Argentina including patients with influenza A or B without risk factors for complications of influenza [35]. Males and non-pregnant females aged 18-64 years with at least one respiratory symptom (cough, sore throat, or nasal symptoms) starting within 48 h before screening between 2012 and 2017 were randomized to receive oseltamivir $75 \mathrm{mg}$ or placebo twice daily for five days. The primary endpoint was the percentage of participants with virus detectable by polymerase chain reaction in nasopharyngeal swab at day three. The first 50 patients randomized were part of a pilot study with frequent study visits to determine the optimal day to assess the primary endpoint. Of the 716 screened, 558 were randomized, of which 501 had confirmed influenza ( $n=246$ oseltamivir; $n=255$ placebo). Baseline demographics were similar between groups and a majority of study patients were Asian (69\%). Out of 449 patients included in the primary endpoint, the oseltamivir group had virus detected on day three in $45 \%$ of patients compared to $57.2 \%$ of the placebo arm (absolute difference $-12.2 \%$; $95 \% \mathrm{CI}-21.4 \%$ to $-3.0 \%$ ). This difference was larger in those enrolled within $24 \mathrm{~h}$ of symptom onset. There was no difference in median time to alleviation of symptoms between groups ( $79 \mathrm{~h}$ oseltamivir group vs. $84 \mathrm{~h}$ placebo). While this study's aim was to evaluate virologic efficacy and it was not powered to detect a difference in symptom duration, it found no difference despite a decrease in viral load. This study has important implications for EM clinicians because it adds to the argument against initiation of oseltamivir in patients without high risk of complication. Previous studies have demonstrated a reduction in symptoms by approximately $24 \mathrm{~h}$, secondary infections (e.g. bacterial pneumonia) and hospitalizations, with the most benefit observed early after symptom onset [37]. However, this benefit is offset by adverse effects like nausea, vomiting, and diarrhea [38]. Based on these findings in 
conjunction with previous studies, we advocate for having an informed discussion with low-risk patients in favor of not initiating treatment for influenza.

Alteplase for Acute Ischemic Stroke in Patients Aged > 80 Years: Pooled Analyses of Individual Patient Data [39] [GRADE 1A].

Intravenous alteplase for stroke with unknown time of onset guided by advanced imaging: systematic review and meta-analysis of individual patient data [40] [GRADE 1A].

Thrombolysis for acute ischemic stroke in the unwitnessed or extended therapeutic time window [41] [GRADE 1A].

IV alteplase is the guideline-endorsed IV reperfusion treatment of acute ischemic stroke (AIS) in patients meeting administration criteria [42]. However, the optimum population and time window for administration continues to be evaluated with recent meta-analyses assessing the use of alteplase in patients $>80$ years of age and two additional meta-analysis evaluating the use of alteplase in an extended or unknown symptom onset.

A pooled analysis of seven randomized trials investigated alteplase $0.9 \mathrm{mg} / \mathrm{kg}(n=3026)$ compared to placebo or open control $(n=$ 3009) for AIS in elderly patients [39]. Patients were divided in subgroups as follows: $\leq 80$ years (alteplase $n=1182$, placebo $=1123$ ), $>80$ years (alteplase $n=518$, placebo $n=510$ ). Outcomes evaluated included good stroke outcome (modified Rankin Scale [mRS] of 0-1 at 90 and 180 days), mortality at $\leq 7$ and $\leq 90$ days, and adverse events including symptomatic ICH and fatal ICH within seven days. For patients $>80$ years, good stroke outcomes were seen more frequently in the alteplase group ( $19.1 \%$ vs. $13.1 \% ; p=0.01$ ) with higher rates of symptomatic ICH $(3.7 \%$ vs. $0.4 \% ; p<0.0002)$ and fatal ICH in seven days (3.5\% vs. $0.4 \% ; p=0.0004)$. There was no difference in seven-day ( $10.6 \%$ vs. $7.8 \%, p=0.13$ ) or 90 -day mortality $(29.5 \%$ vs. $30.2 \%, p=$ 0.84 ). Overall, when comparing AIS patients $\leq 80$ years of age vs. $>80$ years of age, mortality is higher in the older population irrespective of intervention ( $10.2 \%$ vs. $29.9 \%, p<0.0001)$. Of note, several authors were employees and/or consultants for Boehringer who provided funding for this study. The findings suggest that alteplase for AIS in patients $>80$ years of age may improve clinical outcomes and that age alone should not be a barrier to alteplase administration, but rather therapy should be evaluated based on individual risk-benefit.

Two recent meta-analyses evaluated the use of alteplase for AIS with unknown symptom onset or extended therapeutic time window (e.g. $>4.5 \mathrm{~h}$ ) with guidance by advanced imaging. The first used a random effects model and included 4 trials $(n=859)$ that utilized CT or MRI to identify patients with evidence of substantial viable hypoperfused tissue [41]. Advanced neuroimaging techniques included perfusiondiffusion MRI, perfusion CT, or fluid attenuated inversion recovery (FLAIR)-diffusion-weighted imaging (DWI mismatch). The median time for last seen well to symptom recognition ranged from 5.1 to 7.7 $\mathrm{h}$; time from last seen well to alteplase bolus ranging from 7.2 to 10.3 $\mathrm{h}$. The unadjusted analysis found alteplase to be associated with higher three-month favorable functional outcome (OR 1.48; 95\% CI 1.12 to 1.96), three-month functional independence (OR 1.42; 95\% CI 1.07 to 1.90), symptomatic ICH (OR 5.28; 95\% CI 1.35 to 20.68), and complete recanalization (OR 3.29; 95\% CI 1.90 to 5.69). Only three-month favorable functional outcome (OR 1.62; 95\% CI 1.2 to 2.2 ), functional improvement (OR 1.42; 95\% CI 1.11 to 1.81 ) and symptomiatic ICH (OR $6.22 ; 95 \% \mathrm{CI} 1.37$ to 28.26 ) remained significant after adjusting for age and baseline stroke severity. There was no difference in all-cause mortality at three months (OR $1.75 ; 95 \%$ CI 0.93 to 3.29 ).

The second meta-analysis included four studies with 429 patients receiving alteplase and 414 receiving placebo or standard of care [40]. Of note, one trial evaluated $0.6 \mathrm{mg} / \mathrm{kg}$ of alteplase compared to standard of care [43]. Administration of alteplase was associated with a favorable functional outcome at 90 days (OR $1.49 ; 95 \% \mathrm{CI} 1.10$ to 2.03), better functional improvement at 90 days (OR $1.38 ; 95 \% \mathrm{CI} 1.05$ to 1.80 ), and functional independence at 90 days (OR 1.50; $95 \%$ CI 1.06 to 2.12). There was no difference in mortality at 7 days (OR 2.54; $95 \%$ CI 0.78 to 8.32 ), although mortality was higher in the alteplase group at 90 days (OR 2.06; 95\% CI 1.03 to 4.09). Symptomatic intracranial hemorrhage occurred more frequently in the alteplase group (OR 5.58; 95\% 1.22 to 25.50). Image guided therapy for patients with AIS of unknown onset resulted in better functional outcomes at 90 days compared to placebo or standard of care despite an increased risk of symptomatic ICH and 90-day mortality. The two meta-analyses highlight the ever evolving evidence for alteplase administration [40,41]. Regardless, alteplase for AIS should continue to be individualized for each patient weighing both the benefits and risks of therapy.

\section{Conclusion}

This review summarizes key EM pharmacotherapy articles that were indexed in 2020 for the practicing EM clinician. A total of 14 articles were included and their impact on practice was detailed.

\section{Funding/grant}

This research did not receive any additional grants from funding agencies in the public, commercial, or not-for-profit sectors.

\section{Author contributions}

BF and MAR conceived the review topic. DEZ took the lead in combining summaries and writing the manuscript. PS, CSB, BF, and MAR served as senior reviewers and provided critical feedback. All authors performed a literature search, reviewed the identified studies, and contributed to the final form of this manuscript.

\section{Declaration of Competing Interest}

The authors declare that they have no known competing financial interests or personal relationships that could have appeared to influence the work reported in this paper.

BWG, GTH, GWS reports receiving consulting fees from TerSera Therapeutics.

GTH received consulting fees from Kedrion Biopharma, Inc. for participation in a human rabies immune globulin advisory board meeting and writing two manuscripts.

\section{Appendix A. Supplementary data}

Supplementary data to this article can be found online at https://doi. org/10.1016/j.ajem.2021.05.061.

\section{References}

[1] Guyatt GH, Oxman AD, Vist GE, et al. GRADE: an emerging consensus on rating quality and strength of recommendations. BMJ. 2008;336(7650):924-6.

[2] Lindsay P, Schull M, Bronskill S, Anderson G. The development of indicators to measure the quality of clinical care in emergency departments following a modifieddelphia approach. Acad Emerg Med. 2002;9(11):1131-9.

[3] Moskowitz A, Huang DT, Hou PC, et al. Effect of ascorbic acid, corticosteroids, and thiamine on organ injury in septic shock: the ACTS randomized clinical trial. JAMA. 2020;324(7):642-50.

[4] Fujii T, Luethi N, Young PJ, et al. Effect of vitamin C, hydrocortisone, and thiamine vs hydrocortisone alone on time alive and free of vasopressor support among patients with septic shock: the VITAMINS randomized clinical trial. JAMA. 2020 Feb 4;323 (5):423-31.

[5] Marik PE, Khangoora V, Rivera R, Hooper MH, Catravas J. Hydrocortisone, vitamin C, and thiamine for the treatment of severe sepsis and septic shock: a retrospective before-after study. CHEST. 2017;151(6):1229-38. 
[6] Rowell SE, Meier EN, McKnight B, et al. Effect of out-of-hospital tranexamic acid vs placebo on 6-month neurologic outcomes in patients with moderate or severe traumatic brain injury. JAMA. 2020;324(10):961-74.

[7] Roberts I, Shakur H, Coats T, et al. Effects of tranexamic acid on death, vascular occlusive events, and blood transfusion in trauma patients with significant haemorrhage (CRASH-2): a randomized, placebo-controlled trial. Lancet. 2010;376(9734):23-32.

8] The CRASH-3 Trial collaborators. Effects of tranexamic acid on death, disability, vascular occlusive events and other morbidities in patients with acute traumatic brain injury (CRASH-3): a randomised, placebo-controlled trial. Lancet. 2019;394 (10210):1713-23.

[9] Roberts I, Shakur-Still H, Adolabi A, et al. Effects of high-dose 24-h infusion of Tranexamic acid on death and thromboembolic events in patients with acute gastrointestinal bleeding (HALT-IT). Lancet. 2020;395(10241):1927-36.

[10] April MD, Arana A, Schauer SG, et al. Ketamine versus Etomidate and Peri-intubation hypotension: a National Emergency Airway Registry Study. Acad Emerg Med. 2020; 27:1106-15

[11] Mohr NM, Pape SG, Runde D, Kaji AH, Walls RM, Brown 3rd CA. Etomidate use is associated with less hypotension than ketamine for emergency department Sepsis intubations: a NEAR cohort study. Acad Emerg Med. 2020;27:1140-9.

[12] Sagarin MJ, Barton ED, Chng Y-M, Walls RM. Airway management by US and Canadian emergency medicine residents: a multicenter analysis of more than 6,000 endotracheal intubation attempts. Ann Emerg Med. 2005;46:328-36.

[13] Wagner RL, White PF, Kan PB, Rosenthal MH, Feldman D. Inhibition of adrenal steroidogenesis by the anesthetic etomidate. N Engl J Med. 1984;310:1415-21.

[14] Cuthbertson BH, Sprung CL, Annane D, et al. The effects of etomidate on adrenal responsiveness and mortality in patients with septic shock. Intensive Care Med. 2009; 35:1868-76.

[15] Chan CM, Mitchell AL, Shorr AF. Etomidate is associated with mortality and adrenal insufficiency in sepsis: a meta-analysis. Crit Care Med. 2012;40:2945-53.

[16] Edwin SB, Walker PL. Controversies surrounding the use of etomidate for rapid sequence intubation in patients with suspected sepsis. Ann Pharmacother. 2010;44: 1307-13.

[17] Jo SW, Hwang SY, Jo IJ, et al. Ketamine use for endotracheal intubation in severe sepsis and septic shock. Signa Vitae. 2018;14:24-30.

[18] Dewhirst E, Frazier WJ, Leder M, Fraser DD, Tobias JD. Cardiac arrest following ketamine Administration for Rapid Sequence Intubation. J Intensive Care Med. 2012;28: 375-9.

[19] Miller M, Kruit N, Heldreich C, et al. Hemodynamic response after rapid sequence induction with ketamine in out-of-hospital patients at risk of shock as defined by the shock index. Ann Emerg Med. 2016;68:181-8 e2.

[20] Jabre P, Combes X, Lapostolle F, et al. Etomidate versus ketamine for rapid sequence intubation in acutely ill patients: a multicentre randomised controlled trial. Lancet. 2009;374(9686):293-300.

[21] Li B, Zhao H, Zhang J, Yan Q Li T, Liu L. Resuscitation fluids in septic shock: a network meta-analysis of randomized controlled trials. SHOCK. 2020;53:679-85.

[22] Rhodes A, Evans LE, Alhazzani W, et al. Surviving Sepsis campaign: international guidelines for management of sepsis and septic shock: 2016. Crit Care Med. 2017; 45:486-552.

[23] Lamontagne F, Richards-Belle A, Thomas K, et al. Effect of reduced exposure to vasopressors on 90-Day mortality in older critically ill patients with Vasodilatory hypotension: a randomized clinical trial. JAMA. 2020;323(10):938-49.

[24] Lamontagne F, Day AG, Meade MO, et al. Pooled analysis of higher versus lower blood pressure targets for vasopressor therapy septic and vasodilatory shock. Intensive Care Med. 2018;44(1):12-21.

[25] Daya MR, Leroux BG, Dorian P, et al. Survival after intravenous versus intraosseous amiodarone, lidocaine, or placebo in out-of-hospital shock-refractory cardiac arrest. Circulation. 2020;141(3):188-98.
[26] Feinstein BA, Stubbs BA, Rea T, Kudenchuck PJ. Intraosseous compared to intravenous drug resuscitation in out-of-hospital cardiac arrest. Resuscitation. 2017;117: nous

[27] Kawano T, Grunau B, Scheurmeyer FX, et al. Intraosseous vascular access is associated with lower survival and neurologic recover among patients with out-ofhospital cardiac arrest. Ann Emerg Med. 2018;71:588-96.

[28] Mody P, Brown SP, Kudenchuck PF, Chan PS, et al. Intraosseous versus intravenous access in patients with out-of-hospital cardiac arrest: insights from the resuscitation outcomes consortium continuous chest compression trial. Resusication. 2019;134: 69-75.

[29] Granfeldt A, Avis SR, Lind PC, et al. Intravenous vs. intraosseous administration of drugs during cardiac arrest: a systematic review. Resuscitation. 2020;149:150-7.

[30] Panchal AR, Bartos JA, Cabanas JG, et al. Part 3: adult basic and advanced life support: 2020 American Heart Association guidelines for cardiopulmonary resuscitation and emergency cardiovascular care. Circulation. 2020;142:S266-468.

[31] Kudenchuk PJ, Brown SP, Daya M, et al. Amiodarone, Lidocaine or placebo in out-ofhospital cardiac arrest. N Engl J Med. 2016;374:1711-22.

[32] Kim DK, Kim JH, Lee JY, et al. Reappraisal of the treatment duration of antibiotic regimens for acute uncomplicated cystitis in adult women: a systematic review and network meta-analysis of 61 randomised clinical trials. Lancet Infect Dis. 2020;20 (9):1080-8.

[33] Jorgensen SJC, Yeung SL, Zurayk M, et al. Leveraging antimicrobial stewardship in the emergency department to improve the quality of urinary tract infection management and outcomes. Open Forum Infect Dis. 2018;5(6):ofy101. https://doi.org/ 10.1093/ofid/ofy101.

[34] Gupta K, Hooton TM, Naber KG, et al. International clinical practice guidelines for the treatment of acute uncomplicated cystitis and pyelonephritis in women: a 2010 update by the Infectious Diseases Society of America and the European Society for Microbiology and Infectious Diseases. Clin Infect Dis. 2011:52(5):e103-20.

[35] Beigel JH, Manosuthi W, Beeler J, et al. Effect of oral oseltamivir on virological outcomes in low-risk adults with influenza: a randomized clinical trial. Clin Infect Dis. 2020;70(11):2317-24

[36] Ryoo SM, Kim WY, Sohn CH, et al. Factors promoting the prolonged shedding of the pandemic (H1N1) 2009 influenza virus in patients treated with oseltamivir for 5 days. Influenza Other Respi Viruses. 2013;7(5):833-7.

[37] Dobson J, Whitley RJ, Pocock S, Monto AS. Oseltamivir treatment for influenza in adults: a meta-analysis of randomised controlled trials. Lancet. 2015;385(9979): 1729-37.

[38] Rech MA. Oseltamivir for all: should it become an over-the-counter medication for influenza treatment? Pharmacotherapy. 2020;40(3):182-5.

[39] Bluhmki E, Danays T, Biegert G, Hacke W, Lees KR. Alteplase for acute ischemic stroke in patients aged $>80$ years: pooled analyses of individual patient data. Stroke. 2020;51(8):2322-31.

[40] Thomalla G, Boutitie F, Ma H, et al. Intravenous alteplase for stroke with unknown time of onset guided by advanced imaging: systematic review and meta-analysis of individual patient data. Lancet. 2020;396(10262):1574-84

[41] Tsivgoulis G, Katsanos AH, Malhotra K, et al. Thrombolysis for acute ischemic stroke in the unwitnessed or extended therapeutic time window. Neurology. 2020;94(12): e1241-8.

[42] Powers WJ, Rabinstein AA, Ackerson T, et al. Guidelines for the early Management of Patients with Acute Ischemic Stroke: 2019 update to the 2018 guidelines for the early Management of Acute Ischemic Stroke: a guideline for Healthcare professionals from the American Heart Association/American Stroke Association. Stroke. 2019;50(12):e344-418.

[43] Koga M, Yamamoto $\mathrm{H}$, Inoue $\mathrm{M}$, et al. Thrombolysis with Alteplase at $0.6 \mathrm{mg} / \mathrm{kg}$ for stroke with unknown time of onset: a randomized controlled trial. Stroke. 2020;51 (5):1530-8. 


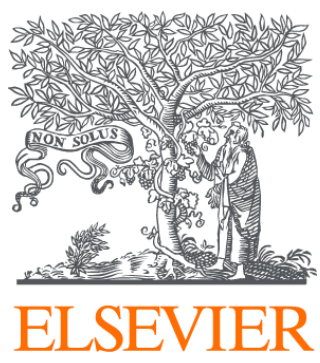

Since January 2020 Elsevier has created a COVID-19 resource centre with free information in English and Mandarin on the novel coronavirus COVID-

19. The COVID-19 resource centre is hosted on Elsevier Connect, the company's public news and information website.

Elsevier hereby grants permission to make all its COVID-19-related research that is available on the COVID-19 resource centre - including this research content - immediately available in PubMed Central and other publicly funded repositories, such as the WHO COVID database with rights for unrestricted research re-use and analyses in any form or by any means with acknowledgement of the original source. These permissions are granted for free by Elsevier for as long as the COVID-19 resource centre remains active. 